\title{
Del tiempo insostenible y del sentido del tiempo en las comunidades kichwa canelos
}

\author{
Rodrigo Martínez Novo
}

En el presente artículo realizamos una aproximación teórica al sentido temporal de las comunidades kichwa canelos de la Amazonia ecuatoriana. Confrontaremos este sentido con la noción temporal que subyace en el discurso moderno del desarrollo sostenible, en un contexto indígena de creciente institucionalización y conflicto. Como veremos, por medio del análisis del discurso y de las metáforas del "delante" y "detrás", la concepción del tiempo kichwa demuestra un carácter analógico y concurrente. Un tiempo colectivo no enajenado, a diferencia del tiempo insostenible moderno occidental.

PALABRAS CLAVE: tiempo, desarrollo sostenible, comunidades kichwa, delante, detrás, metáfora

\section{Unsustainable Time and Time Sense in Kichwa Canelos Community}

This article provides a theoretical approach to the temporal sense of the Kichwa Canelos communities in the Ecuadorian Amazon. It will be held by confronting this sense with the temporal notion that underlies the modern discourse of sustainable development in an indigenous context of increasing institutionalization and conflict. As we will see through discourse analysis and the "before" and "behind" metaphors, the Kichwa concept of time is based on analog and concurrently character. A time not alienated, such as modern western unsustainable time.

KEYWORDS: time, sustainable development, Kichwa communities, in front of, behind, metaphor 


\section{INTRODUCCIÓN}

$\mathrm{T}$ an natural como hablar del tiempo climático parece poderse hablar también del tiempo social. Hemos dado por sentado que se puede decir de él con tal autonomía y objetividad, que no es extraño que sea ahora él quien marque las horas y diga de nosotros. Su legitimidad reside en la capacidad que se le atribuye como dominio externo para plasmar nuestra propia experiencia: ya sea este dominio regularmente seccionado en forma de línea recta - como la historia-, ya sea en forma circular - como el reloj de agujas-. Pero el análisis que nos atañe muestra que el tiempo no siempre vive fuera como una categoría obviada, dada o programada, y de la que se puede hablar sin más. Tiene matices cambiantes, tempos distintos cuyas cualidades no dependen sólo de la velocidad o la frecuencia desplegadas sobre la recta. Por ello estamos obligados a hablar de él, al menos, como de un "sentido" y no tanto como un fenómeno de la expehablar de un tiempo ya contado o cuantificado, nos interesan la formas en que de ese tiempo se piensa y se habla — se da por descontado- para dar sentido a los hechos que involucran a una comunidad de hablantes. Entendemos también, en coherencia con Evans (2004: 212), que existe una "matriz" amplia de sentidos del tiempo que impide su asunción como universal en una única vía comprensiva. ${ }^{1}$

Conviene alertar que esta falta de universalidad no sumerge nuestro análisis en la relatividad más

\footnotetext{
${ }^{1}$ La alusión al tiempo que realizaremos en este trabajo se aleja de la interpretación durkheimiana como representación colectiva de un modo de cuantificación o periodización (Durkheim, 1968). Compartimos el carácter colectivo del tiempo, mas no -en acuerdo con Alfred Gell (1992) - que éste se configure necesariamente como un esquema de referencia cuantitativo, métrico o cronológico de un periodo: grupo de puntos localizados paradójicamente en el espacio del tiempo, como un calendario. Ello se demuestra en la utilización de referentes temporales no cuantitativos como el "antes" o "después" indicados por Kant, o incluso en el uso de las metáforas de "delante" y "detrás" que analizaremos en este artículo.
}

absoluta, en el aislamiento artificial del cognitivismo cultural o en el del fetichismo material o artefactual. No es producto aislado de sí mismo como singularidad simbólica ni lo es de un uso tecnológico específico. Nuestro enfoque entiende el sentido del tiempo como un proceso cambiante cuya narración se establece principalmente con base en un contexto conflictivo moderno, como en el que viven los kichwa canelos. Es en él donde se producen controversias conceptuales que muestran en qué medida se está configurando y recreando este sentir temporal. Nos centraremos, por tanto, en destacar los contrasentidos temporales que surgen en un ámbito mediado por el discurso del desarrollo sostenible. ${ }^{2}$ Con base en las primeras premisas expuestas nos aproximaremos al contexto conflictivo de las comunidades amazónicas para sugerir los contrasentidos que surgen en torno al desarrollo sostenible y su sentido temporal. En segundo lugar, nos adentraremos en estos contrasentidos mediante el análisis de las características del sentir temporal de los kichwa canelos comunitarios.

\section{IDENTIDADY CONTEXTO: DE VUELTA CON EL DESARROLLO, REVUELTAS CONTRA EL DESARROLLO}

Runa - persona, humano- es el nombre con el que se denominan a sí mismos los kichwa canelos. La identidad a la que hacen alusión está relacionada con procesos de transculturización y etnogénesis que involucran a varias culturas asentadas históricamente en el bosque tropical de la Amazonia ecuatoriana. Según señalan algunos autores, su origen se

\footnotetext{
${ }^{2}$ Clifford Geertz (1968) ha mostrado en su aproximación al tiempo del calendario balinés cómo el contexto influye en su conceptualización. Conviene añadir desde un enfoque discursivo, como el que aquí emprendemos, que el contexto no puede desligarse de las relaciones de poder que lo implican ni de la pragmática en que se evidencia (Alonso, 1999). Asumimos esta perspectiva a pesar de que supera las expectativas y la extensión del presente artículo.
} 
debe a los diversos grupos étnicos que pudieron confluir en los núcleos misioneros de esta región buscando refugio de las políticas institucionales coloniales, como la encomienda o la tributación (Restrepo y Cabrejas, 1998). ${ }^{3}$ En la actualidad sus comunidades se distribuyen intermitentemente desde la ciudad del Puyo hacia el este, por los cauces de los ríos Pastaza, Curaray, Villano y Bobonaza. De forma general desarrollan un estilo de vida adaptada al bosque tropical basado en la caza, la pesca, la recolección y una agricultura de subsistencia o chacra, que combinan con pequeñas transacciones monetarias. En el ámbito de lo social destaca una estructura basada en las relaciones de parentesco que gira en torno al ayllu, grupos de parientes por consanguinidad, afinidad y ritualidad. A pesar de la permanencia actual de este sistema de organización en el interior de la selva, las luchas por salvar sus lugares de vida contra las actuaciones del desarrollo - espacialmente acuciantes a partir de la mitad del siglo $\mathrm{xx}$ - les ha llevado a convivir con nuevas formas de institucionalidad externa. Como señala Reeve, las comunidades aglutinadas en torno a federaciones legalmente reconocidas tratarían no sólo de mejorar la eficiencia de sus reivindicaciones ante el Estado, sino también de defenderse de las enormes presiones lanzadas desde los centros nacionales e internacionales para ser "desarrollados y productivos" (Reeve, 2002).

Prueba de ello son los numerosos conflictos y levantamientos que han involucrado a las comunidades de la Amazonia ecuatoriana a finales del siglo pasado. En 1990 ocurrió el denominado "levantamiento indígena”, en el que participaron 40000 indígenas de todo el país. Mediante la paralización del suministro de materias primas a la capital, consiguieron plantear al Estado el reconocimiento de sus derechos ancestrales colectivos y territoriales,

\footnotetext{
${ }^{3}$ Según las recientes normas gramaticales kichwas, la letra ' $\mathrm{k}$ ' remplaza a la ' $q$ ' y a la 'c', regla que aplicaremos en la escritura de este documento, salvo en la palabra canelos, que hace referencia a la misión católica del mismo nombre.
}

así como la declaración del Ecuador como un Estado plurinacional (Ojeda, 2005). Las conversaciones con el gobierno se interrumpieron drásticamente en la negociación de uno de los puntos del "mandato" planteado por la organización de pueblos amazónicos Kichwa Aashuar y Shiwiar de la Organización de Pueblos Indígenas de Pastaza (OPIP). En el controvertido punto se reclamaba la firma de los "Acuerdos de Sarayacu", un documento elaborado por la comunidad amazónica de Sarayacu — canelos kichwa- a raíz del proceso de lucha contra la incursión de la compañía petrolera Arco Oriente en sus territorios. La negativa del gobierno a proseguir con las conversaciones se hizo firme y llevó a estos pueblos amazónicos a protagonizar en 1992 el segundo levantamiento indígena. En una marcha histórica de más de 400 kilómetros desde la región amazónica hasta Quito, se congregaron alrededor de 2000 indígenas encabezados por la OPIP, con una reivindicación territorial que tuvo como consecuencia el reconocimiento legal de 1115574 hectáreas de tierra. En esta época las comunidades y sus nuevas confederaciones e instituciones comenzaron a elaborar estrategias para "planificar y racionalizar" las principales líneas de acción que regirían los espacios de propiedad recién adquiridos. Los enfoques que orientaron estas líneas se imbricaban dentro de un contexto de fracaso de los modelos de desarrollo tradicionales y favorecieron la emergencia de movimientos sociales en torno a la etnicidad y el ecologismo (Fontaine, 2003). De esta forma y por mediación de la opIP, los canelos kichwa han acogido en su territorio un cúmulo de proyectos y planes orientados hacia un "desarrollo alternativo", que se refleja en el uso de conceptos como "desarrollo autónomo integral" u otros más conocidos, como "desarrollo sostenible". No obstante y pese a su presunta condición de alternativos, todos estos intentos se han enfrentado a un gran número de dificultades y errores que dan testimonio de los límites que imponen todavía los diversos usos e interpretaciones del concepto de "desarrollo".

\footnotetext{
${ }^{4}$ Véanse ejemplos prácticos en Martínez (2010).
} 


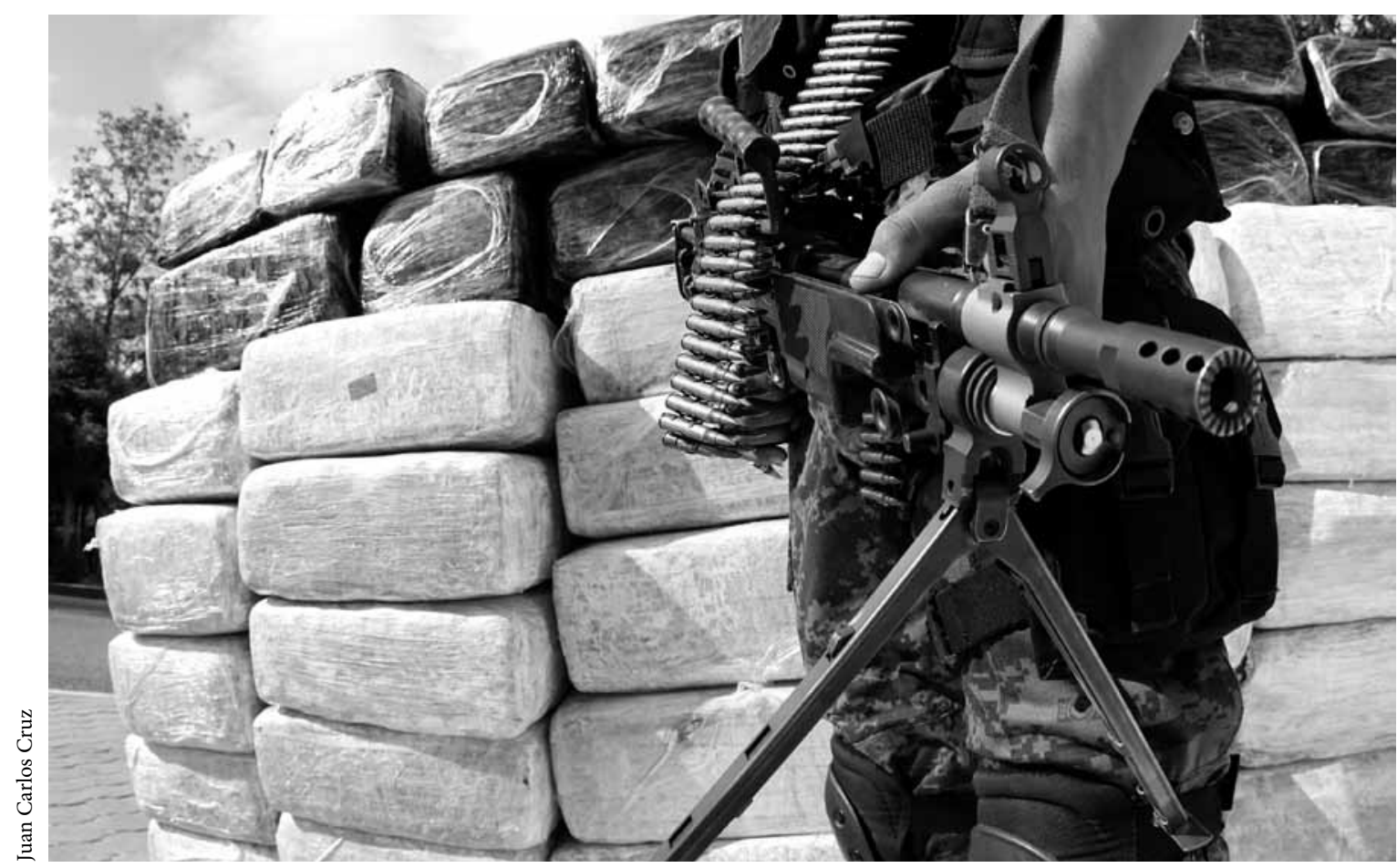

Toneladas de mariguana y drogas sintéticas aseguradas por el Ejército durante los operativos contra el narcotráfico en Sinaloa, 2011.

El mismo año un colectivo de kichwas y mestizos elaboró el "Plan Amazanga", que presentaba el enfoque conceptual y filosófico de los kichwa de Pastaza sobre el manejo de los recursos naturales. Este documento exponía de manera clara el antagonismo con el difundido concepto de "desarrollo sostenible". Las principales aportaciones críticas se hacían evidentes a través de uno de los términos que el plan recogía y sobre el que establecían las bases conceptuales de su propuesta. Nos referimos al sumack kawsay o "bien vivir". Más allá de las referencias que incluye dicho documento institucional, uno de nuestros informantes comunitarios nos muestra la importancia de este concepto en la vida kichwa y cómo se opone en esencia a todo el significado desarrollista:

Sumack kawsay simplemente significa que vivimos en el presente, que nos levantamos día a día y vivimos felices. No hablan del desarrollo sostenible en las comunidades, sólo viven en el presente y no hablan de futuro. Vivir tranquilo, en paz, sin hacer daño a nadie, eso es nada más (informante 3 ).

El fragmento evidencia un claro antagonismo con el desarrollo sostenible que a primera vista puede parecer extraño: si bien el desarrollo había sido claramente denostado por los movimientos indígenas, la bondad del nuevo epíteto "sostenible" parecía poder ajustarse a las representaciones modernas indígenas de lo que Astrid Ulloa denomina "nativo ecológico". Pero más allá la idealización de un "buen salvaje" que en la práctica muestra sus contradicciones - cae por su propio peso-, la persistencia del concepto institucional está relacionada con un blindaje implícito en su propia estructura semántica, aparentemente inofensiva. El tipo de figuras conceptuales construidas con base en la unificación de los opuestos - como desarrollo y sostenibilidad - con el fin de hacer un lenguaje 
inmune a la contradicción - como la habida entre ecologistas y desarrollistas - se consigue "a costa de vaciarlos de contenido y de acentuar su carácter tautológico" (Naredo, 2006: 145). No obstante, la vacuidad y la ambigüedad no son asépticas, pues posibilitan que los actores en posiciones de poder puedan erigirse en legitimadores de su significado. Si bien la sostenibilidad hace de complemento indispensable en dicha construcción, hay que admitir que la fuerza que imprime el desarrollo en su significado ascendente y lineal no puede ser sostenido. Son entonces los sentidos más sólidos del desarrollismo los que continúan presentes como tendencia en las acciones que involucran a cualquiera de las posiciones que usan el concepto.

Nuestro informante hace explícitas las diferencias que devuelven la bondad sostenible a su significado desarrollista, puntualizaciones concretas que son objeto de reflexión en este artículo. Si contraponer "vivir bien" con desarrollo ya es sintomático del tipo de valoración que les suscita este término, también lo es analizar en qué diferencias se centran para hacer explícito esto. En concreto, el tiempo parece estar muy involucrado en estas diferenciaciones. Su alusión a esa manera de vivir bien en el presente no sólo es la forma de poder compartir con nosotros el sentido de sumak kawsay, sino también la forma de evidenciar la existencia de un contrasentido que realza precisamente lo que los distingue, lo que el desarrollo sostenible y los desarrollistas no practican: vivir en el presente significa no vivir para el futuro ni dejar que el tiempo pase sin más. Supone una noción que, en la medida en que se aleja de nuestra concepción, genera - en términos de Michaell Agar (2002) - un choque cultural y lingüístico.

Como señala Ernest García a partir de la Conferencia de Río de 1992: "todo lo que existe bajo la luz del sol está llamado a desarrollarse de modo sustentable" (García, 2002: 146), pero sus preceptos y, en lo que aquí respecta, el tiempo no pueden darse por obviados. Pues el tiempo del desarrollo sostenible, a pesar de los sutiles cambios aportados con su innovadora figura, no ha podido sostener - evitar- ese tiempo que tiene sus cimientos en una tradición cultural muy concreta, que con abuso podemos catalogar de "occidental": "aquella en la que se conjuga la concepción griega abstractiva y ávida de regularidades, y esa otra hebrea lineal y orientada, como corresponde a un pueblo nómada que, instalado en la historia, avanza hacia un echaton" (Lizcano, 1992). Muy diferente es el sentido del tiempo kichwa canelos, en el que no se reproduce una comprensión cronológica y consecutiva de los "tiempos que pasan" ni de un futuro inédito - o como repetición del presente- al cual dirigirse de forma programada o planificada.

\section{DEL SENTIDO DEL TIEMPO DE LOS KICHWA CANELOS}

Para aproximarnos al sentido kichwa canelos del tiempo hemos identificado en su forma de hablar - con gran dificultad - tres modos de "pseudotiempo" que responden con imprecisión a lo que en castellano podría describirse como "pasado", "presente” y “tiempo mítico". Según Whitten (1987) y Reeve (2002), los tiempos a los que nos referimos son: kallari — pasado-, que se enlaza a múltiples presentes o kunan y un espacio atemporal con gran cantidad de elementos míticos, denominado unai, que atraviesa el resto de esferas temporales a través de relatos y visiones. El pasado o kallari es un proceso de acontecimientos, testimoniados y transmitidos de generación en generación hasta nuestros días, ocurridos antes de la llegada de los misioneros a las comunidades y que viven en el presente a través de la tradición oral. Los relatos suelen describir guerras, migraciones y detalles alusivos a la desaparición de grupos étnicos - como los zaparos, de gran importancia en la zona-. Nuestros informantes hablan así sobre el kallari: ${ }^{5}$

\footnotetext{
${ }^{5}$ Los extractos que mostramos reproducen la forma en que han escrito, dicho o traducido las frases kichwa nuestros informantes indígenas amazónicos, por lo que seguramente pueden incurrir
} 
El kallari corresponde a la historia antes de la llegada de los misioneros españoles y de los caucheros al Amazonas (informante 1) (cursivas mías).

El kallari, nuestros antepasados vivieron de caza, pescas y agricultura, eran más o menos nómadas, buscaban dónde había abundante caza y expertos guerreros que combatían con la tribu shuar por las mujeres (informante 2).

Antes - en el kallari- había cacería y pesca pero están acabándose ya. No hay peces como había antes. Antes había unos chamanes y han dañado a la madre de los bagres - un pez que proporciona abundante alimento a las comunidades - en la laguna. Antes lanzábamos el anzuelo y sacábamos bagres grandes, pero se han ido llevando todo. Los chamanes zaparos tenían un arma con la que luchaban entre ellos, llamada grejón, para matar. Y peleaban, por lo que estaban haciéndose daño. Y desde esa temporada se han ido bajando el número de bagres (informante 3 ).

A pesar de las interferencias lingüísticas, que al hablar en nuestro idioma redundan en una aparente comprensión cronológica - como "la historia" o el "antes"-, observamos que los acontecimientos descritos no pueden datarse de forma concreta ni situarse sobre los periodos medibles de la línea cartesiana. Por el contrario, se encuentran en el saco inconmensurable del kallari, aquel tiempo antes de la llegada del tiempo determinado - "el de los misioneros" - de donde se extraen los acontecimientos en el momento de su relato para cobrar valor por sí mismos, por su propio peso significativo, $y$ no por la posición que juegan o el orden consecutivo en el que se inscriben. En el caso del último fragmento (informante 3), lo importante es que los zaparos chamanes luchaban entre sí y eso influyó en la merma de los peces. El acontecimiento, lejos de constituirse como uno más de los hitos u objetos

en faltas gramaticales tanto del kichwa unificado como del castellano. Hay que tener en cuenta que el kichwa amazónico y el serrano presentan algunas diferencias entre sí y con el idioma oficializado, además de que ambos han sido originariamente orales. Por ello nos hemos esforzado en respetar, por encima de todo, la representación sonora de lo dicho a través de lo escrito. de la recta histórica, deviene en el presente para ser interpretado por la subjetividad de quien lo refiere: porque debido precisamente a esas guerras, en la actualidad no hay peces como había antes.

Otro de los tiempos identificables, el presente - kunan para los kichwas-, adquiere sentido a través del pasado. De hecho, no puede existir en abstracto si no incluye algún aspecto tradicional (Whitten, 1987: 68). La sabiduría y el conocimiento sobre el kallari compone la cualidad del presente, le da forma hasta constituirlo y establecer diferencias actuales en función de él. Los ancianos en contacto directo con la sabiduría de sus antepasados viven en kunan rucuguna - presente de los antiguos o los antiguos de hoy-, mientras que los jóvenes de la comunidad viven en kunan malato - presente de los jóvenes- (informante 4). El cúmulo de experiencias y conocimientos sobre prácticas culturales consideran una existencia determinada. Implica que los presentes, los comunitarios, se definen en función de ellas y son kichwas en la medida en que ese pasado cultural los conforma con mayor o menor intensidad. Sobre estas dos esferas de pasado y presente interfiere y actúa el unai. Éste es un espacio/tiempo indiferenciado, cronotópico, ${ }^{6}$ que se utiliza para hacer referencia a una época tan primordial que se pierde más allá del pasado: un tiempo remoto antes del tiempo. Está repleto de aspectos adimensionales, seres mitológicos con apariencia entre humana y animal, espíritus supais, etcétera:

unaimanda ñucanchic puricmaranchic sacha ucuta, tucuita ricusha pariuglla sacha supaigunahuand

[Desde un inicio - unai manda- nosotros caminábamos dentro de la selva viendo todo junto a los dioses de la selva] (informante 4).

Debido a las características adimensionales del unai, su presencia es ubicua, puede deslocalizarse y mezclarse con el resto de tiempos identificables,

\footnotetext{
${ }^{6}$ Con cronotopo nos referimos a la unidad indisociable que se da en la conciencia mítica entre el espacio y el tiempo (Torop, 2002).
} 
tanto en el presente como en el pasado. Desde el presente, los runa pueden penetrar en su universo por medio de rituales, cantos, sueños y visiones inducidas por plantas, como la ayahuasca - especie de Beniseriopsis - o el huanduj - especie de datura-. Al entrar establecen contacto con seres antiguos en un espacio donde es especialmente habitual la transformación y el intercambio de cualidades entre humanos, plantas y animales:

Precisamente esta noche tomé ayahuasca y el chamán me estaba conversando sobre las almas de los seres que quedan en la selva. Cuando un ser humano muere, se convierte en un animal, en una boa, un tigre, un venado o un mono lanudo, y no se muere, sigue viviendo.

Ellos dicen que en Unión Base - comunidad cercana al Puyo-, allá adentro están todos los animales que existían. Por ejemplo, nuestros chamanes, donde ahora hay pastizales, simplemente no podemos ver nada pero allí están todos los animales que antes vivían, los tapires, los primates y los dioses que les protegen a ellos (informante 5) (cursivas mías).

Cuando existían nuestros padres guando, en ese tiempo existía una planta allicauguando - una datura- para convertirse en puma. Yo puedo ser también tigre. Yo he tomado la planta dos veces. Cuando yo estoy aquí en la comunidad, los wawas - niñoshan escuchado en la pica cuando iban a la escuela que el tigre rugía (informante 6).

El unai no sólo es parte de ese presente desde el cual se accede, sino que se encuentra de manera recurrente en los testimonios relacionados con acontecimientos pretéritos. La intromisión mitológica en estos relatos acompaña el sentido que se les otorga desde el presente. Al hacerlo difumina las fronteras temporales, las torna confusas y casi atemporales.

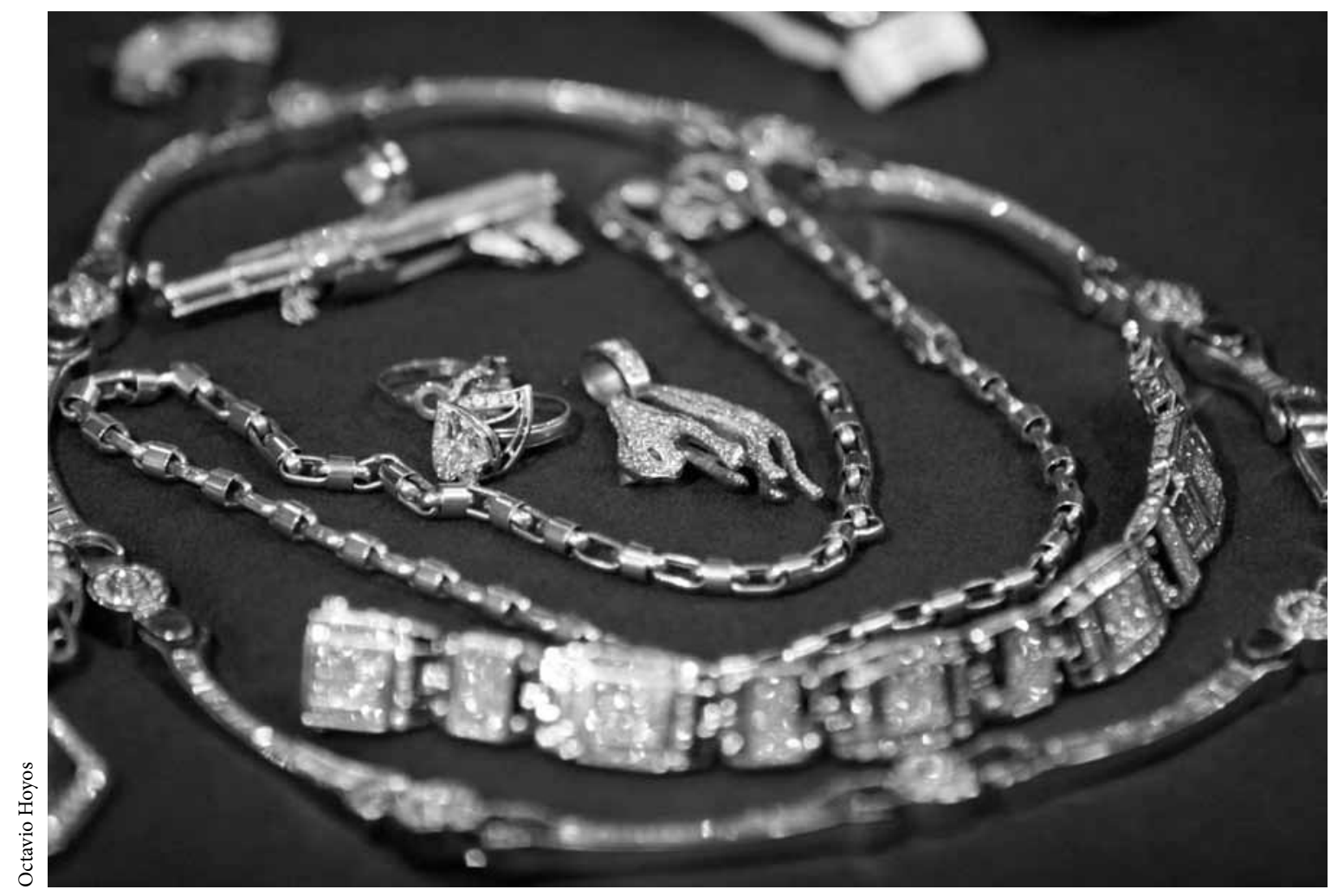

Presentación de decomiso de joyas por integrantes de la Marina de México a una banda de narcotraficantes en Sinaloa, 2009. 
Veamos ahora en un relato del pasado con fuertes elementos míticos la función de su presencia:

- ¿Que bebían para poder transformarse en boa? (investigador).

-Era un proceso de ayuno con plantas que sólo ellos conocían y donde absorbían los poderes de los supais —espíritus de la selva - y así de la Madre Tierra. Por eso se escuchaba la existencia del Atacapi [boa mitológica de dos cabezas] en el Río Tiriri. Cerca de esa zona, existe un cerro cuyo nombre es Atacapi. Ese cerro se mueve, ese cerro definitivamente es Atacapi, está poseído por los poderes de los supais.

En ocasiones durante la noche se produce una claridad inusual como si fuera iluminado por luces. Si entras en esta zona, Atacapi te absorbe, te come. Todos los seres que osan aproximarse $\mathrm{o}$ atravesar esta laguna desaparecen misteriosamente.

Cierto día un chamán muy experto del Perú, quien tenía la capacidad de convertirse en boa, pasó la frontera del Aguarico hasta llegar al Cerro Atacapi. Luego de un largo proceso de ayuno quiso contactar con los supais del cerro para poder aumentar su poder. Pero no lo consiguió y desapareció absorbido por el poder del Atacapi. El chamán permaneció adormecido en el estómago del Atacapi, por una semana, pero pudo esconder un cuchillo de chonta [madera muy densa que se utiliza para fabricar herramientas] muy afilado. Un día, cuando el Atacapi dormía tomando el sol, el chamán advirtió que se había tumbado en la arena del río y decidió salir desgarrando el estómago del Atacapi. Pero antes de escapar cortó las venas principales del corazón para asegurarse que iba a morir. Inmediatamente murió y su cuerpo comenzó a absorber como una esponja el agua de la laguna. La laguna del Tiriri se convirtió en una pequeña poza (informante 7) (cursivas mías).

Para dar respuesta a nuestra pregunta, el narrador empieza relatando acontecimientos del pasado - "era un proceso de ayuno"-, pero enseguida introduce un componente mitológico a través de un ser característico del unai: el "Atacapi”. Este intenso solapamiento de las dos dimensiones - pasado y unai- permite dar sentido a un proceso geomorfológico que comienza tiempo atrás y culmina en la actualidad: el paso de una laguna, la del Tiriri, a una pequeña poza. Sin embargo, el conocimiento así ganado es atemporal (Lévi-Strauss, 1964), ya que la cronología o linealidad se ve interrumpida por la intromisión en el pasado de un mito temporalmente deslocalizado. Es por ello que los seres que pueblan la selva y los escenarios sagrados con los que conviven se desprenden de esta dimensión que especialmente los ancianos se encargan de reavivar en sus conversaciones. Hace las veces de un puente que une y homogeneiza o, como dice uno de nuestros informantes, se utiliza para hablar del "desde hace tiempo" pero para "cuentos instantáneos", integrando en una sola estructura lo que para nosotros sería un antes y un ahora.

Mediante la presencia ubicua del unai difícilmente pueden identificarse delimitaciones nítidas, mucho menos en lo que concierne a la existencia de una frontera clara del tiempo futuro: de ese acontecer inédito que proyecta a largo plazo unos acontecimientos como si estuvieran dados. Quizá el lenguaje runa no refleja este vocablo explícito, porque, como nos dijo uno de nuestros entrevistados en la selva, simplemente "no hay". Encontramos el concepto más parecido a esta idea en la "visión esporádica" del sumack muskui - excelente sueño-y sólo en tanto que ésta conlleva una percepción lejana de un acontecimiento. Nuestros informantes lo definen así:

Son visiones que la gente indígena tenemos que tener para ir a cazar, pescar, conseguir peladas, ir a la guerra, para no accidentarse $\mathrm{u}$ otro cualquier cosa, eso es el sumak muskui (informante 2).

Ninguno de nosotros soñaba a futuro, si no ya nos hubiéramos preparado. Los sabios tomaban guando $\mathrm{y}$ ayahuasca y veían lo que iba a pasar a futuro y llegaban a tener ese sueño en visión. Pero no hay ninguna idea del camino que se debe seguir, solamente guiarse de la persona más anciana de la comunidad y de su sabiduría (informante 3) (cursivas mías).

Lo que a priori podría considerarse una concepción análoga al futuro es en el mejor de los casos una visión - "un sueño"- que se produce desde el pasado, desde la sabiduría de los ancianos. Las 
Gráfica 1. Visión esporádica del sumack muskui

Tiempo mítico: unai

Futuro esporádico: sumack muskui

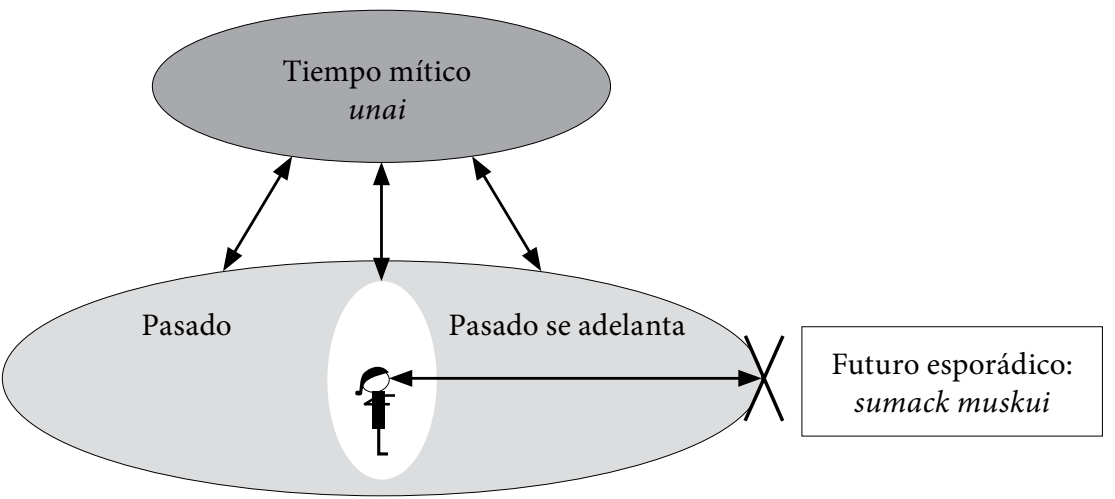

Fuente: Elaboración propia.

premoniciones que advierte el sueño del sumack muskui están sugeridas por las inquietudes del individuo que accede a este sueño desde el presente y transcurren dentro de una estructura mítica en la que de nuevo se revela la presencia del unai. Como ocurre en muchas tribus nómadas, el futuro posible, el que no deviene un sueño, está en el "horizonte del presente percibido". Ése es el denominado "por venir" al que alude Bourdieu en sus estudios de la Cabilia en Argel (Bourdieu, 1963: 61). Más allá de él, el futuro deviene un sueño esporádico que al contrario que la promesa del desarrollo no se construye sobre la novedad absoluta, sino que se tiñe de pasado (en la gráfica 1 tratamos de representarlo).

A pesar de lo esgrimido, algunos autores llegan a encontrar en esta cosmovisión una noción de linealidad. Whitten - referente fundamental en el estudio de los kichwas amazónicos- argumenta esta idea con base en la creencia de los kichwa de que la mayor parte de su sabiduría se perdió durante un periodo concreto que divide su concepción del pasado en dos tiempos diferenciados, dotándola de una trayectoria casi cronológica: los tiempos antiguos y los tiempos de los abuelos. Whitten (1987: 69) denomina "tiempo de destrucción" al periodo intermedio, ya que coincide con la época del caucho
(Reeve, 2002; Colier, 1968; Casement, 1912; Taussig, 1984; citados por Whiten, 1987: 69), de invasiones de esclavos y guerras que mermaron la población amazónica:

Gráfica 2. Tiempo lineal según Whitten (1987)

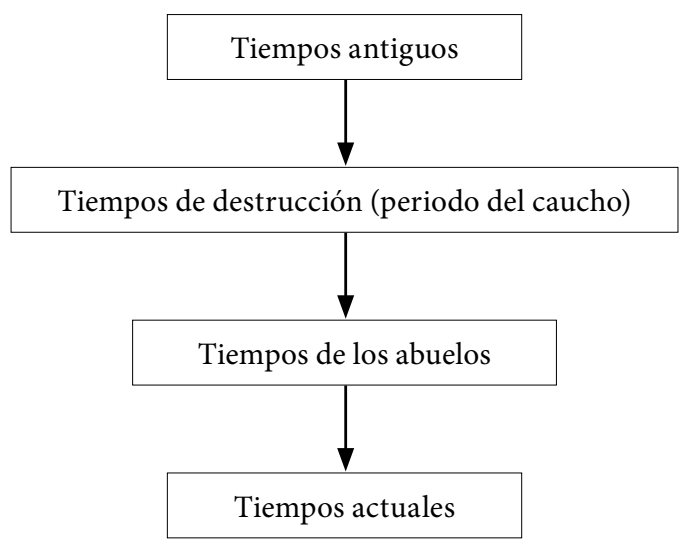

Fuente: Elaboración propia.

En efecto, debemos admitir que existe un enlace o una continuidad lineal entre el presente y el pasado en el sentido que apunta Reeve de que el conocimiento obtenido a partir del unai - tiempo mítico- y del 
kallari trasciende, se transmite y se transforma. También es cierto que existe un pasado reciente para los kichwa - tras la devastadora irrupción de los españoles, el fin de los tiempos antiguos-, durante el cual se ha potenciado el contacto con la educación formal de las escuelas rurales y, por tanto, comienzan a utilizarse términos históricos cronológicos. Sin embargo, no podemos admitir que la percepción del tiempo pueda ser claramente compartida como lineal en tanto secuencia de intervalos de magnitudes regulares, ya que el tiempo mítico impide su subordinación a un ideal geométrico cuantificable. No se trata de un sentir que encaja en sistemas o espacios temporales, antes bien se basa en eventos concretos que dan forma al tiempo. Lo concreto define el tiempo del mismo modo en que lo señala Evans-Pritchard en su estudio sobre los nuer: el ciclo de actividades determina el ciclo conceptual del calendario y no al contrario (EvansPritchard, 1992). De igual modo, el futuro no existe en abstracto como un espacio-tiempo a rellenar, sino que deviene en una proyección interpretada desde lo ya vivido. En este transcurrir sinuoso ${ }^{7}$ el pasado no pasa, sino que se hace momento, deja huella.

\section{ANALOGÍA, ESPACIOY TIEMPO}

El sentido del tiempo concreto que hemos descrito se interpreta con mayor claridad desde el modo de pensamiento que Lévi-Strauss (1964) denominó

\footnotetext{
${ }^{7}$ La visión cíclica no es extraña a otras sociedades fuera del ámbito descrito como indígena en la actualidad: "Esa representación simbólica de lo cíclico, de que todo en el cosmos nace, muere, renace, es fuerte en las sociedades primitivas, pero está presente también en las pequeñas comunidades tradicionales de pequeños agricultores itinerantes, de pescadores y de recolectores que todavía viven al sabor de los ciclos naturales y en un complejo calendario agrícola" (Diegues, 2000: 56). Por otro lado, en referencia a los kichwa de la sierra, Faller y Cuéllar (s/a) señalan que no es un acontecer exactamente cíclico, más bien "sinuoso", como la trayectoria que sigue el sol por el cielo.
}

bricoleur: aquel en el que todos los momentos acontecidos y atravesados por la sustancia mítica se disponen como una despensa estática alrededor del individuo que los nombra. El tiempo cultural y colectivo se compone de múltiples piezas de puzzle, de cristales rotos que encajan con el presente a medida que se les hace momento. Cada individuo recolecta aquel acontecimiento transmitido - sembradoen la red de relaciones comunitarias, que por su relevancia se recuerda o puede ponerse en práctica. Como veíamos en el kallari, lo pasado no está calculado por medio de una longitud cronológica que a medida que se aleja respecto del presente parece menos accesible, ipues ya ha pasado! En su lugar se recurre a la analogía para contraponer los acontecimientos sucedidos en el tiempo.

Las comparaciones, sin embargo, utilizan formas de relación, expresiones que de alguna forma lo sitúan entre sí temporalmente. Son metáforas del espacio que permiten posicionar unos acontecimientos en relación con otros transportando la experiencia del espacio al tiempo. Aunque las metáforas espacio-temporales son universales para algunos (Núñez, 2003), las representaciones kichwa de esta experiencia presentan diferencias significativas respecto de las formas comunes con que suelen caracterizarse. ${ }^{8}$ De forma genérica, los autores que tratan este tema desde la literatura lingüística y cognitiva

\footnotetext{
${ }^{8}$ Los estudios del lenguaje y las ciencias cognitivas que entienden el sentido del tiempo como una relación del tiempo con el espacio son herederas de los a prioris kantianos, en los que el espacio y el tiempo son categorías cognitivas independientes y universales preexistentes. Respecto del lenguaje, si bien para autores como Lakoff el espacio y el tiempo no se han concebido como categorías preconceptuales o prelingüísticas (Lakoff, 1987), sí se han asumido como universales, como propias de cualquier lenguaje, lo cual conviene relativizar, dado que, como se demuestra en estudios cognitivos, el tiempo no siempre se referencia en relación con el espacio (Boroditsky, 2000), no todas las culturas utilizan estas relaciones metafóricas - como los amondawa de la Amazonia (Zinken et al., 2006) - y no todos los ámbitos del Occidente moderno son proclives a este uso: véanse las nuevas metáforas surgidas en la "vida on-line" (Browdle y Gentner, 1995 y 1999). Es importante destacar, como lo hace Jullien (2005), que si bien las metáforas del espacio en relación con el tiempo son abundantes y dispersas, lo son especialmente en el llamado Occidente.
} 
señalan dos formas de representar metafóricamente el tiempo y sus eventos en relación con el espacio (Clark, 1973; Lakoff y Johnson, 1980; Mc Taggart, 1908). La primera remite a las denominadas metáforas que representan el "ego en movimiento" y que tienen su reflejo en expresiones como "nos acercamos ya a las vacaciones". En este caso la flecha temporal atraviesa al individuo desde el pasado, por el presente y hacia el futuro. El individuo se dirige hacia delante - "hacia las vacaciones" - como quien camina hacia un hito espacialmente situado, dejando atrás espacial y temporalmente todo lo caminado. La segunda corresponde a las que aluden al tiempo en movimiento, caracterizadas por expresiones como "queda poco tiempo para que lleguen las fiestas", en las que el tiempo atraviesa de forma inversa al individuo, desde el futuro, por el presente y hacia el pasado. En éstas el tiempo transporta los eventos hacia el individuo, acercándolos por delante y alejándose hacia el pasado por detrás. En ambas modalidades el pasado está detrás y el futuro delante, y también en ambas, a pesar de sus denominaciones, el ego juega un papel fundamental, siendo referenciado en uno u otro sentido en relación con el tiempo (Moore, 2006). Menos atención se ha prestado a aquellas metáforas en las que el tiempo y el observador son más estáticos y en las que las expresiones reflejan referencias espaciales que sitúan unos tiempos respecto de otros. A partir de este tipo de representaciones relativas - no deícticas o no egocéntricas- los kichwa referencian espacio-temporalmente sus eventos concretos. Veamos ahora las características de estas representaciones en relación con el modelo relativo castellano.

\section{El espacio del tiempo: metáforas de "delante" y "detrás" en el tiempo kichwa}

Como señalan Moore (2006) y Haspelmath (1997), hay una tendencia a representar relaciones dicotómicas en expresiones de tiempo no deícticas, como delante/antes y detrás/después, en numerosos idiomas: en inglés, before/after; en latín, ante/post; en tammil, munnaale/pinnaale; en maorí, mua/muri; en mandarín, quián/hòu; en japonés, mae/ato, o en madagasco, alhoa/aoriana. Conviene no confundir el "delante" y "detrás" con el "antes" y "después", pues su uso diferenciado deriva en cualidades discursivas nada despreciables. Por ejemplo, en el modelo relativo castellano es común hacer alusión a un "antes" o a un "después" en el devenir de la recta espacio-temporal: podemos decir "antes de ayer" o "pasado mañana" — después de mañana-del mismo modo que decimos que alguien nació "antes" o "después" que otro, primero o segundo. Estos conceptos se utilizan a menudo para traducir otros conceptos del lenguaje kichwa que no son, sin embargo, homólogos. Nos referimos al uso de las metáforas espaciales ñawpak - delante- y washa —detrás- en kichwa amazónico.

-Shut puma ñuka ñawpacpi aw [Hay un puma delante de míl.

-Shut puma ñuka washay aw [Hay un puma detrás de mí] (informante 2).

Del mismo modo en que un objeto o un animal - un puma- pueden situarse "delante" o "detrás" en el espacio respecto de la persona u otro objeto, los acontecimientos pueden relacionarse entre ellos o con el tiempo presente del individuo mediante esta metáfora. Sin embargo, este "delante" y "detrás" imprime un sentido distinto al antes o después. ${ }^{9} \mathrm{De}$ hecho, pueden advertirse intuitivamente ciertas diferencias cuando leemos la traducción literal de este concepto en una frase del kichwa - utilizando el

\footnotetext{
${ }^{9}$ Que nuestros informantes hablen de "antes" o "después" cuando traducen las frases kichwas al castellano no significa que éste sea su sentido explícito. Más bien, la traducción está enfocada hacia un receptor para el que la utilización del "delante" o "detrás" no adquiere sentido en las relaciones semánticas de su lenguaje común, ése que los kichwa están acostumbrados a escuchar. Por ello consideramos su traducción una adaptación, de otro modo, muy significativa.
} 
término delante-, y cuando lo hacemos a continuación pero sustituyendo el término por uno con sentido en nuestro idioma - anteriormente-:

-Ñaupakmi rimacgaraunchic cutis: delante/anteriormente sabíamos contar esto.

- Ñaupak causashcai ashca pumagunata ricucgaranchic: delante/anteriormente había más pumas.

-Cai taripasha musiarisha ricunchi ñucanci yachaigunata, cunan pachai, washa pachai, mutsurishcagunata pactachin: así damos a conocer con cuidado nuestra sabiduría o conocimientos de este tiempo y para el tiempo detrás/de después, para nuestros jóvenes.

-Washa shamungarau: vendrá detrás/después (informante 4).

Las diferencias son significativas. En el caso del uso del "antes" y el "después" con sentido en castellano, su significado interviene en la cualidad movible del acontecer, estableciendo los márgenes. Cuando leemos o escuchamos que "anteriormente había más pumas" se nos está informando que entre antes y hoy los pumas han ido desapareciendo. Desde fuera del propio acontecer de los pumas se encapsula un intervalo de tiempo que, sin embargo, no niega ni la inercia ni el movimiento de los sucesos acotados. Una noción muy próxima a aquella con la que Aristóteles describía el tiempo: "el número del movimiento respecto al antes y al después” (Fraser, 1992: 26).

La inercia de los propios acontecimientos apunta a un "antes" que vaticina lo que dejará el "después". Como si se tratara del paso de la mirada apresurada de un lector por las líneas de un nuevo libro, lo leído se sitúa por lo que va a ser, o por lo que ya nunca será. Un tiempo que ya ha pasado dirigido hacia un después que desde la lejanía se promete escrito: lo que "vendrá después" puede abandonar en su firmeza "lo que antes había”. Lo leído en esa primera lectura ilusionante queda impreso y ya se puede "pasar página". A partir de entonces todo parecerá una relectura de lo mismo. Los acontecimientos quedarán marcados por el índice, un orden sin reinterpretaciones aparentes. Sólo el uso del "antes" y el "después" en expresiones que sitúan dichos sucesos nos recuerda que esa cronología sólida es paradójicamente el resto dejado por una inercia constante. Es un tiempo constante, propio de un universo con sus propias leyes - como el universo-máquina newtoniano- en el que los acontecimientos parecen narrarse por sí solos. Un tiempo que en su autonomía y solidez nos narra y que más que ser leído parece leernos.

Por el contrario, desde el sentido kichwa canelos en el modelo relativo el uso del "delante" y el "detrás" nos aproxima a la cualidad estática de los eventos. Lo que está delante - los pumas - todavía no ha pasado ni pasa de forma incontestable y lo que viene detrás no es un después huidizo. La dualidad espacial con que se establecen referencias temporales no realza su tendencia a escaparse en su propio movimiento enajenado, sino que lo hace dependiente del momento que le llama y lo sitúa: lo que viene "detrás" no niega lo que está "delante", no lo abandona, simplemente lo coloca a disposición, como en una despensa. El movimiento está en boca de quienes lo nombran y no forma parte de una inercia ajena o idealizada.

\section{El espacio del tiempo: metáforas de “delante" y "detrás" en el modelo deíctico del idioma castellano}

También es posible encontrar en castellano metáforas del tiempo relacionadas con el "delante" o el "detrás" en el tipo de expresiones denominadas deícticas. Por ejemplo: "tienes un gran futuro por delante", "tienes que enfrentar tu futuro" o "he dejado muchas cosas tras de mí" - en el pasado-. Las frases, tomando como referencia al individuo, o ego, sitúan el futuro delante y el pasado detrás. Lo ejemplificado establece las siguientes relaciones:

Gráfica 3. Modelo deíctico

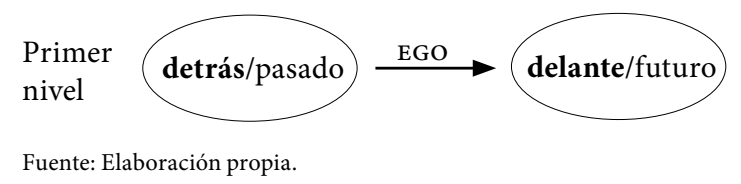


Este tipo de expresiones no se registran ni en el kichwa de la Sierra (Faller y Cuéllar, s/a) ni en el kichwa canelos de la Amazonia. Aunque en kichwa no se utilizan referencias en torno al modelo del "ego", sí existen, como hemos visto, representaciones cercanas al modelo relativo que muestran sus contrasentidos al compararlas.

\section{Gráfica 4. Modelo relativo kichwa}

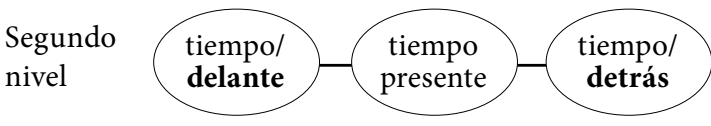

Fuente: Elaboración propia.

Dado que ambos modelos están en distintos niveles de significación, no podemos atribuir al concepto de tiempo kichwa un sentido linealmente inverso al occidental, donde el pasado estaría delante y el futuro detrás del individuo. O por lo menos no encontramos las mismas evidencias que dicen encontrar algunos estudios similares en relación con los aymaras (Gifford, 1986; Núñez y Sweetser, 2006)..$^{10}$ En el primer nivel, el tiempo occidental se caracteriza por imponer desde fuera una inercia hacia delante, un tempo en el que se diluyen los momentos del sujeto que avanza desde dentro. A corto plazo es un tiempo que corre calculado y repetido: la hora de entrada a la fábrica, la hora de salida y el tiempo libre. A largo plazo es un tiempo programado y obviado sin contar con el pasado, como si fuera un lector que olvida al comienzo de cada capítulo aquello que leyó antes, lo que quedó detrás.

Por el contrario, en el segundo nivel el lenguaje kichwa se centra en lo acontecido o en el momento presente para traer a su memoria todo eso que está en la parte de adelante del libro, lo ya leído o pasado.

\footnotetext{
${ }^{10}$ En algunos estudios sobre la visión del tiempo en los aymaras se asegura que cuando se insta a los miembros ancianos de esas poblaciones a situar el pasado levantan el brazo indicando hacia delante (Gifford, 1986; Núñez y Sweetser, 2006). Esta evidencia gestual con referencia al cuerpo no ha sido registrada en las comunidades kichwas de la Amazonia.
}

Desde esta posición compara aquellas anécdotas del relato que vienen - mientras lee- de atrás del libro. Se distancia por tanto del modelo del "ego" seguramente por no representar un observador que desde dentro del río-tiempo anuncia los baches a los que le arroja la corriente. Sin embargo, tampoco estamos de acuerdo en que el tiempo se relacione en kichwa, como apuntan Faller y Cuéllar (s/a), desde una posición completamente externa al río. Más bien nos aproximamos a la perspectiva cognitiva de Kevin Ezra Moore, que en relación con el caso aymara destaca que no se trata ni de un modelo egocéntrico ni de uno relativo completamente, sino de la unión entre ambos marcos de referencia para situar los acontecimientos. En nuestro caso y tras el análisis expuesto esto se traduce en que el "delante" y el "detrás" suscriben un modelo estático que hace difícil establecer los límites del propio cauce temporal. El sujeto se encuentra en una posición intermedia entre dentro y fuera, y a partir de ésta hace condensar sobre sus momentos los eventos temporales que recoge en forma de relación espacial.

\section{ALGUNAS RE-CONSIDERACIONES}

Como hemos visto, el sentido del tiempo kichwa, "esa forma de vivir en el presente sin hablar del futuro" al que hacía alusión nuestro primer informante, deviene en un contra-sentido del tiempo moderno que implica la concepción del desarrollo sostenible: ese tiempo lineal, en progreso constante, donde el pasado desaparece, se agota y el futuro, aunque se desconoce, se presume sustentable, programable y planificable. Un tiempo "para las generaciones futuras", como bien anuncia el famoso Informe Brundtland, que le dio luz. El tiempo analizado es muy distinto. En las dimensiones del tiempo kichwa se enreda una concepción mítica en la que los acontecimientos cobran importancia por analogía y donde, en lugar de ser la linealidad periódica la que marque los eventos, son éstos los que marcan su paso. Destaca también la ausencia de esa 


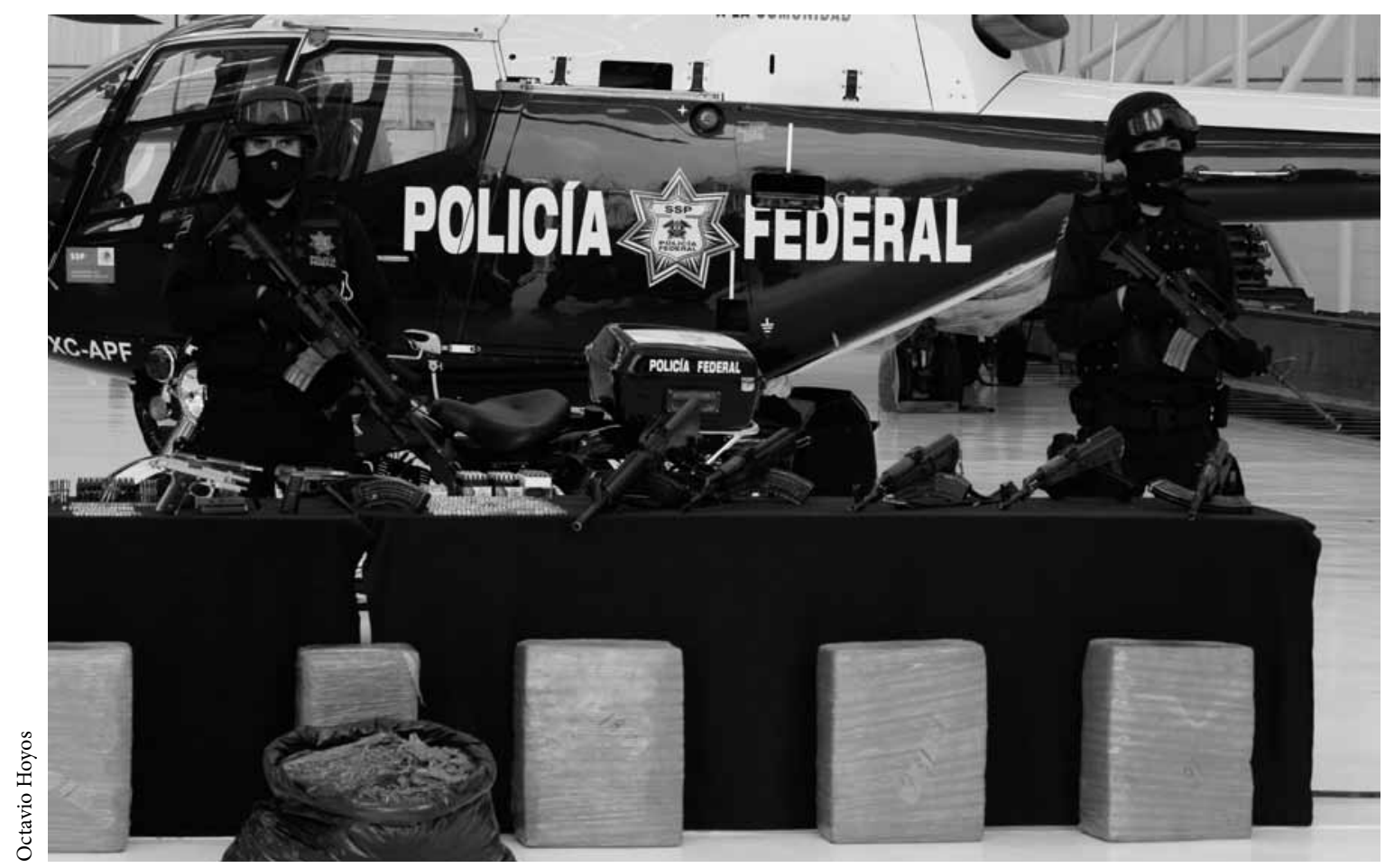

Armas y drogas decomisadas presentadas en las instalaciones de la Policía Federal, 2009.

inercia con que la flecha de un tiempo ajeno arrastra al sujeto: dejando el pasado atrás, pasando por el presente y siempre ante un futuro al cual dirigirse.

La visión desarrollista que se sostiene en esta concepción se forma sobre la metáfora biológica del cambio o la mutación constante (Esteva, 1996). Señala un tiempo sumergido en su propia inercia natural y autónoma, sin tomar en consideración la importancia que tienen en él los sujetos que lo nombran y el pasado que, más que pasar, lo constituye. Haciendo uso del mismo lenguaje biológico, pero desde una interpretación más cercana a la concepción kichwa analizada, podríamos considerar que todo desarrollo biológico es, a su vez, la repetición de un desarrollo precedente inscrito genéticamente en sus células dentro de un contexto o un suelo con diferentes características que lo condicionan. Entonces lo ocurrido, a pesar de los cambios, es más el regreso cíclico del pasado que la reconstrucción inédita del futuro (Morin, 1980).
Con base en esto queremos sugerir, a expensas de posteriores trabajos, que en los nuevos contextos indígenas institucionales de gestión del territorio la planificación en aras de cualquier desarrollo es proclive a negar esta re-interpretación. ${ }^{11}$ La planificación permite justificar la asunción de un tiempo constante en cuya inercia se abandona el presente, en aras del futuro que está delante. De hecho, para que el

\footnotetext{
${ }^{11}$ En este sentido, cabe considerar que no son los discursos de los kichwas comunitarios, sino las instituciones, la administración, las federaciones indígenas y los mediadores indígenas ventrílocuos los que continúan reproduciendo el desarrollismo en sus discursos y no de forma inocua. Como señala Santamarina a propósito de los estudios de Brosius (1999) y Escobar (1995) sobre los procesos de institucionalización: aunque "la institucionalidad puede ser vista, en algunos aspectos, como positiva (el propósito de la conservación medioambiental), es necesario atender a los procesos por los cuales estas instituciones inscriben y naturalizan ciertos discursos, ya que al tiempo que proponen alternativas para la preservación, excluyen otras posibilidades, privilegian a ciertos actores y marginan a otros" (Santamarina, 2008: 175).
} 
futuro lejano no se vea impregnado de un pasado mítico -o devenga en un sueño como el de los kichwa comunitarios-, para que esté delante situado más allá de ese horizonte presente "por venir", el futuro debe pre-verse, pro-yectarse, plan-i-ficarse. Este por venir ciego, innovador, repleto de pre-fijos que arrastran hacia delante la medida espacial del tiempo tiene la misión de convertir los imprevistos en pre-vistos. Olvida sin embargo que la utilidad de las previsiones depende de que los im-previstos estén también pre-destinados a ocurrir. Una vez que se le ha dado cuerda al reloj, una vez pro-gramado el tiempo, tanto el tiempo marcado como el plan orquestado se justifican por sí mismos, ordenan los actos. Nadie recuerda quién da vueltas a las manecillas de esos "tiempos que corren", o si el desarrollo es legítimo o no. Por ende, también es difícil saber si los im-previstos son su causa o el resultado de una profecía autocumplida que beneficia a ciertos intereses.

A partir de aquí, el riesgo de lo acontecido no está en la incertidumbre del futuro a planificar o en su complejidad inconmensurable, sino en el hecho de que la solución y el problema compartan el mismo espacio delante, como dos caras de la misma moneda. Quizá pueda comprenderse también que se acabe produciendo aquello que se pretendía solucionar, como nos señala Lizcano (1995) respecto de las soluciones que otorga el desarrollo a la pobreza. Se corre el riesgo de que el perro se muerda la cola y de que los conflictos modernos puedan ser justificados en la apariencia que adquieren de ser un producto de sí mismos. Una autopoiesis planificada en la cual los poderes son anónimos y las víctimas culpables.

\section{Referencias}

Agar, Michael, 2002, Language Shock. Understanding the Culture of Conversation, Perennia, Nueva York.

Alonso, Luis Enrique y Javier Callejo, 1999, "El análisis del discurso: del postmodernismo a las razones prácticas”, en Revista Española de Investigaciones Sociológicas, núm. 88, pp. 37-75.
Bergson, Henri, 1910, Time and Free Will: An Essay on the Immediate Data of Consciousness, S. Sonnenschein \& Co., The Macmillan Co., Londres y Nueva York.

Boroditsky, Lera, 2000, "Metaphoric Structuring: Understanding Time through Spatial Metaphors", en Cognition, vol. 75, pp. 1-28.

Bourdieu, Pierre, 1963, "The Attitude of the Algerian Peasant Towards Time”, en Julian A. Pitt Rivers (ed.), Mediterranean Countrymen, Mouton, París, pp. 55-72.

Brosius, Peter, 1999, "Anthropological Engagements with Environmentalism", en Current Anthropology, vol.40, núm. 5, pp. 207-309.

Browdle, Brian y Dedre Gentner, 1995, “The Career of Metaphor", ponencia, Psychonomics Society, Los Ángeles.

— 1999 , "Metaphor Comprehension: From Comparison to Categorization", ponencia, XXI Annual Meeting of the Cognitive Science Society, Vancouver.

Clark, Herbert, H., 1973, "Space, Time, Semantics, and the Child", en Timothy E. Moore, Cognitive Development and the Acquisition of Language, New York Academic Press, Nueva York.

Diegues, Antonio Carlos, 2000, El mito moderno de la naturaleza intocada, Abya Yala, Quito.

Durkheim, Émile, 1968, Las formas elementales de la vida religiosa, Schapire, Buenos Aires.

Escobar, Arturo, 1995, The Making and Unmaking of the Third World, Princeton University Press, Estados Unidos.

Esteva, Gustavo, 1996, "Desarrollo", en Wolfgang Sachs (ed.), Diccionario del desarrollo. Una guía del conocimiento como poder, Proyecto Andino de Tecnologías Campesinas, Lima.

Evans-Pritchard, Edward Evan, 1992, Los nuer, Anagrama, Barcelona.

Evans, Vyv, 2004, The Structure of Time: Language, Meaning and Temporal Cognition, John Benjamins, Ámsterdam.

Faller, Martina y Mario Cuéllar, s/a, "Metáforas del tiempo quechua", en línea: <personalpages.manchester.ac. uk/staff/martina.t.../Faller-Cuellar.pdf $>$, consultado el 10 de diciembre de 2009.

Fontaine, Gillaume, 2003, El precio del petróleo: conflictos socioambientales y gobernabilidad en la Región Amazónica, Facultad Latinoamericana de Ciencias Sociales, Instituto Francés de Estudios Andinos, Quito.

Fraser, Julius, 1992, "El muro de cristal. Ideas representativas sobre el tiempo en el pensamiento occidental", en Archipiélago: Cuadernos de Crítica de la Cultura, núms. 10-11, pp.17-54. 
García, Ernest, 2004, Medioambiente y sociedad: la civilización industrial y los límites del planeta, Alianza, Madrid.

Geertz, Clifford, 1988, La interpretación de las culturas, Gedisa, Barcelona.

Gell, Alfred, 1992, The Anthropology of Time. Cultural Constructions of Temporal Maps and Images, Berg, Oxford.

Gifford, Douglas, 1986, "Las metáforas temporales en aymara y quechua”, en Winak, núm. 2, pp. 4-19.

Hall, Edward, 1983, The Dance of Life. The Other Dimension of Time, Doubleday, Nueva York.

Haspelmath, Martin, 1997, "From Space to Time: Temporal Adverbials in the World's Languages", en Lincom Studies in Theoretical Linguistics, Lincom Europa, Munich.

Jullien, François, 2005, Del tiempo, Arena Libros, Madrid.

Lakoff, George, 1987, Women, Fire and Dangerous Things: What Categories Reveal about the Mind, University of Chicago Press, Chicago.

- y Mark Johnson, 1980, Metaphors we Live By, University of Chicago Press, Chicago.

Lévi-Strauss, Claude, 1964, El pensamiento salvaje, Fondo de Cultura Económica, Madrid.

Lizcano, Emmanuel, 1992, "El tiempo en el imaginario social chino", en Archipiélago: Cuadernos de Crítica de la Cultura, núms. 10-11, pp. 59-68.

1264 1995, "Para una crítica de la sociología de la pobreza”, en Archipiélago: Cuadernos de Crítica de la Cultura, núm. 21, pp. 13-16.

Martínez, R., 2010, “Atravesando el espejo del desarrollo sostenible: una aproximación sociológica al bien vivir de las kichwas canelos", trabajo de investigación, Universidad de Valencia, Valencia.

Mc Taggart, John, 1908, “The Unreality of Time”, en Mind, núm. 17, pp. 457-474.

Moore, Kevin Ezra, 2011, "Ego-perspective and Field-based Frames of Reference: Temporal Meanings of Front in Japanese, Wolof, and Aymara”, en Journal of Pragmatics, núm. 43, pp. 759-776.

Morin, Edgar, 1980, "El desarrollo de la crisis del desarrollo", en Cándido Mendes (comp.), El mito del desarrollo, Kairós, Barcelona.
Naredo, José Manuel, 2006, Raíces económicas del deterioro ecológico y social. Más allá de los dogmas, Siglo XXI, Madrid.

Núñez, Rafael, 2003, “Conceptual Structures and Cultural Variation. Metaphorical Spatial Construals of Time in Aymara", ponencia, Department of Cognitive Science, University of California, San Diego.

y Eve Sweetser, 2006, "With the Future Behind Them, Convergent Evidence From Aymara Language and Gesture in the Crooslinguistic Comparison of Spatial Construals of Time", en Cognitive Science, núm. 30, pp. 1-49.

Ojeda, Lautaro, 2005, "Contexto económico y social de la propuesta de autonomía indígena”, en Liliana Almeida, Nidia Arrobo y Lautaro Ojeda, Autonomía indígena, frente al Estado nación y a la globalización neoliberal, Abya Yala, Quito.

Reeve, Mary Elizabeth, 2002, Los quichuas del Curaray. El proceso de formación de la identidad, Abya Yala, Quito.

Restrepo, Marcus y Antonio Cabrejas, 1998, Canelos: cuna de pastaza, Casa de la Cultura, Puyo.

Santamarina, Beatriz, 2008, "Antropología y medioambiente. Revisión de una tradición y nuevas perspectivas de análisis en la problemática", en Revista de Antropología Iberoamericana, vol. 3, núm. 2, pp. 145-173.

Torop, Peeter, 2002, "Intersemiosis y traducción intersemiótica”, en Cuicuilco. Revista de la Escuela Nacional de Antropología e Historia, t. 2, vol. 9, núm. 25, pp. 13-42.

Ulloa, Astrid, 2001, El nativo ecológico: movimientos indígenas y medio ambiente en Colombia, en línea: <http:// www.antropologia.com.br/arti/colab/a26-aulloa.pdf $>$.

Whitten, Norman, 1987, Etnicidad y adaptación de los quichuas hablantes de la Amazonia Ecuatoriana, Abya Ayala, Quito.

Zinken, Jörg et al., 2006, "When Time is not Space: The Social and Linguistic Construction of Time Intervals and Temporal Events Relation in an Amazonian Culture", en Language and Cognition, vol. 3, núm.1, pp. 137-169. 